\title{
Wie soll man der Opfer von Gewalt und Krieg gedenken?
}

\author{
Aus Anlass einer Publikation der katholischen Kirche in Deutschland \\ Von JoAchim KöHLER
}

Bereits in mehrerer Auflagen liegt ein Martyrologium, eine Sammlung von Biografien deutscher katholischer Opfer von Gewalt und Krieg des 20. Jahrhunderts, vor, ein opulent ausgestattetes, zweibändiges Werk, das von der Deutschen Bischofskonferenz in Auftrag gegeben und finanziert wurde ${ }^{1}$. Man wird sich fragen, warum dieses Werk in einer landesgeschichtlichen Zeitschrift angezeigt und ausführlich besprochen wird. Eine Antwort gibt Joachim Kardinal Meisner, der Erzbischof von Köln, der in seiner Eigenschaft als Vorsitzender der Liturgiekommission der Deutschen Bischofskonferenz das Vorwort geschrieben hat. Plausibel ist die Antwort zunächst nur für Insider der katholischen Kirche, denn er spricht von einer Notwendigkeit, Männer und Frauen zu verehren, die im vergangenen Jahrhundert als „Zeugen für Christus“ ihr Leben geopfert hätten. Zugleich fordert er die katholische Kirche in Deutschland auf, an der Jahrhundert- und Jahrtausendwende Bilanz zu ziehen. Offensichtlich sind für den Kardinal die „Zeugen für Christus“ ein Aktivposten in der Jahrhundert-Bilanz der katholischen Kirche in Deutschland. Auch dieser Hinweis ist nicht ohne Weiteres zu verstehen. Der Kardinal klärt auch das auf, in dem er sich überrascht zeigt, „wie viele Christen gerade in Deutschland mit den gottlosen Systemen des Nationalsozialismus und Kommunismus aus ihrer Treue zum Evangelium heraus in Konflikt geraten sind“. Und er fügt hinzu: „Die katholische Kirche braucht sich ihres Weges durch diese Bedrängnisse nicht zu schämen. Natürlich gibt es auch immer wieder Schwachheit und Versagen ... Wir haben allen Grund, für die Positivseite dieser Bilanz Gott zu danken“.

Meisner beruft sich auf ein Apostolisches Schreiben des Papstes Johannes Pauls II. vom 10. November 1994 (Tertio millennio adveniente), in welchem der Papst die einzelnen Ortskirchen innerhalb der Weltkirche bittet, „einmal die Martyrer des zu Ende gehenden Jahrhunderts aufzulisten“, um sie zu verehren. Denn:

1 Zeugen für Christus. Das deutsche Martyrologium des 20. Jahrhunderts, hg. von Helmut Moln im Auftrag der Deutschen Bischofskonferenz, 2 Bde., 4. vermehrte u. aktualisierte Aufl., Paderborn 2006. 
„Die Verehrung der großen Vorbilder unseres Glaubens sichert eine wirkliche geistliche Demokratie in der Kirche, indem wir in den Problemen der kirchlichen Gegenwart die Lösungsversuche der Generationen gläubiger Menschen vor uns mit in die Waagschale werfen“2 ${ }^{2}$. Papst und Kardinal wollen „den kommenden Generationen eine wirkmächtige Ermutigung an die Hand geben“. Wer soll das verstehen?

Im Mittelalter waren es die Heiligenviten und Heiligenlegenden, mit denen die Verehrung der Heiligen angefeuert wurde. Mit Hilfe des Mediums „Legende“ wurde die herrschende Meinung der Kirche propagiert. Legenden dienten der Erbauung, Pädagogisierung und der Disziplinierung der Gläubigen. Deshalb trat in ihnen die Lehre der Kirche stärker zutage als die historische Wahrheit. Auf diesem Hintergrund taucht die Frage auf, welche Funktion hat das Martyrologium heute? Wie verhalten sich die Berichte der Leiden und Opfer jener Menschen zur historischen Wahrheit? Wie ist der Hinweis auf die „wirkliche geistliche Demokratie in der Kirche“, die durch die Verehrung der Blutzeugen sichergestellt wird, zu verstehen?

Lassen wir erst einmal die Absichtserklärungen der Einleitung auf sich beruhen. Sie werden den heutigen, aufgeklärten Menschen kaum verleiten, in einen Martyrerkult zu verfallen. Schauen wir uns zuerst die Dokumentation an, die Auflistung jener Menschen, die den Terrorsystemen des Bolschewismus und des Nationalsozialismus zum Opfer gefallen sind, und zwar in der territorialen Auswahl bezogen auf die Länder des deutschen Südwestens, die den Lesern dieser Zeitschrift besonders von Interesse sein dürften. Auf diese Weise können wir uns wenigstens einen Teil der im Gesamtwerk aufgelisteten Opfer etwas genauer anschauen und die Umstände ihres Todes - vielleicht - verstehen. Vielleicht gelingt es, auf die Fragen, die uns beim Lesen der Einleitung in den Sinn gekommen sind, eine Antwort zu geben - oder auch nicht. Einzig eine Frage wird stehen bleiben, nämlich ob es sinnvoll ist, eine derartige Dokumentation zu erstellen.

In vier Gruppen werden die Martyrer oder Blutzeugen, und zwar Männer und Frauen, eingeteilt:

A. Blutzeugen aus der Zeit des Nationalsozialismus (1933-1945), S. 1-906 und [seit 2001 neu ermittelte Blutzeugen], S. 1244-1297. Da diese Namen nach Diözesen aufgelistet werden, ist es einfach, Angehörige der südwestdeutschen Diözesen Freiburg und Rottenburg-Stuttgart zu eruieren.

B. Blutzeugen aus der Zeit des Kommunismus (ab 1917), S. 909-992 und S. 12991316. Da es sich um Gebiete handelt, in denen der Kommunismus herrschte, können Blutzeugen aus dem Südwesten nur über Geburtsorte ermitteltet werden.

C. Reinheitsmartyrien (20. Jahrhundert), dabei handelt es sich um Kinder oder Jugendliche, die nur über ihre Herkunftsorte gefunden werden können, und schwerpunktmäßig um „schutzlose Ordensschwestern und Frauen gegen Ende des

2 Alle Zitate bisher sind dem Vorwort von Joachim Kardinal Meisner des zu besprechenden Werkes (wie Anm. 1, Bd. 1, S. XXXIf.) entnommen. 
Zweiten Weltkrieges“, also um Frauen aus den ehemaligen deutschen Ostgebieten, S. 995-1083 und S. 1314-1344.

D. Blutzeugen, Männer und Frauen der Missionsorden (20. Jahrhundert), werden nach ihrer Ordenszugehörigkeit aufgelistet, S. 1085-1242 und 1346-1380. „Opfer“ aus dem südwestdeutschen Raum können wiederum nur über ihren Geburtsort oder über den Standort ihres Heimatklosters im Südwesten ausfindig gemacht werden.

In der Gruppe A: „Blutzeugen aus der Zeit des Nationalsozialismus“ wird das Territorialprinzip, d.h. Gliederung nach den deutschen Diözesen, angewandt. Allerdings ist man überrascht, dass nicht die regionalen Strukturen der Jahre 1939 bis 1945 zugrunde gelegt wurden, in der alle Personen in ihrem Leidens- und Todeskampf verortet waren, sondern die Einteilung der Bistümer zur Zeit der Wiedervereinigung von Bundesrepublik und Deutscher Demokratischer Republik mit den Struktur- und Grenzveränderungen der Nachkriegszeit. Die ehemaligen deutschen Ostgebiete werden nicht mehr zur Bundesrepublik Deutschland gezählt, sondern als Visitaturen, also als virtuelle Territorien (Breslau, Danzig, Ermland, Schneidemühl, Glatz, Sudetenland) aufgelistet. Das mag aus heutiger Sicht politisch korrekt sein, historisch ist es nicht. Denn viele der Blutzeugen gehörten ehemals deutschen Diözesen an, die heute in Polen oder Russland liegen. Aus politischer Rücksicht kann man offenbar nicht erwarten, dass Deutsche, die zum Beispiel in Schlesien ihr Martyrium erlitten, heute in Polen als Heilige verehrt werden.

Innerhalb der Gruppe A werden die Blutzeugen in Priester und Laien unterschieden. Obwohl alle Menschen im Tod gleich sind, obwohl das Zweite Vatikanische Konzil die Kirche als „wanderndes Gottesvolk“ beschrieben hat und die Dokumente des Konzils die Laien nicht mehr als „Objekte“ der Seelsorge beschreibt, sondern als Subjekte ernstnimmt, wird die Kluft zwischen Priester und Laien betont, gemäß dem Kirchenbild des 19. Jahrhunderts. Damals wurde die Kirche als eine "Gesellschaft von Ungleichen“ definiert.

Aus der Erzdiözese Freiburg werden 13 Priester (Alois Beichert, Adolf Bernhard, Alois Brugger, Heinrich Feuerstein, Franz Anton Fränznik, Michel Gaisert, Max Graf, Josef König, Otto Meckler, Max Joseph Metzger, August Ruf, Anton Spies und Willibald Strohmeyer) und drei Laien (Reinhold Frank, Ewald Huth und Richard Kuenzer) genannt. Die Diözese Rottenburg ist nur mit einem Laien, dem Staatpräsidenten Eugen Bolz, vertreten. Berücksichtigt man die Herkunft der Personen, so müssen innerhalb dieser Gruppe A Priester und Laien anderer Diözesen aufgezählt werden. Das sind: Pfarrer Bernhard Heinzmann, geboren in Böhmenkirch, Kr. Göppingen, der Kriegsdienstverweigerer Josef Ruf aus Hochberg, Kr. Saulgau, der Reichtagsabgeordnete Otto Gerig, geboren in Rosenberg/ Baden, und der Münchner Universitätsprofessor Kurt Huber, der seine Jugend in Stuttgart verbrachte.

Ordensmänner dieser Gruppe werden nach ihrer Ordenzugehörigkeit aufgelistet, so dass sie nur über den Geburtsort und/oder über das Kloster, dem sie ange- 
hörten, dem südwestdeutschen Raum zugeordnet werden können: P. Athanasius (August) Gerster OSB aus Dogern, Kr. Waldshut, P. Eugen (Ernst Reinhard) Hiestand OSB vom Kloster Beuron, P. Marcellus (Adolf) Higi OSB aus Bierlingen bei Rottenburg, P. Alfred Delp SJ aus Mannheim, P. Alois Grimm SJ aus Külsheim, der Pallottiner P. Albert Eise aus Oeffingen bei Stuttgart und der Pallottiner Br. Johannes Leodegar Kremer aus Mannheim. Ordensfrauen aus dem südwestdeutschen Raum sind in dieser Gruppe nicht vertreten, es sei denn, man zählt die Karmelitin Edith Stein hinzu, die enge Beziehungen zum Kloster Beuron pflegte.

Personen der Gruppe B: „Blutzeugen aus der Zeit des Kommunismus“ werden eingeteilt in Russlanddeutsche und Donauschwaben, sie alle sind auch in diesen Gebieten geboren. Nur zwei Personen kommen ihrer Herkunft nach aus Deutschland, einer davon ist der Beuroner Benediktiner Adalbert (Karl) Graf Neipperg, der in Schwaigern bei Heilbronn aufgewachsen ist und erster Abt des Klosters Neuburg bei Heidelberg war. Aus dem Laienstand ist nur eine einzige Frau dieser Gruppe zugeordnet, alle anderen Personen sind Priester.

Eine gewisse Konzentration in der Darstellung lässt sich in der Gruppe C: „Reinheitsmartyrien im 20. Jahrhundert“ feststellen. Dieser Gruppe werden acht Schicksale „Schutzloser weiblicher Jugendlicher" vorangestellt, die in den Jahren 1905 bis 1969 im Alter von elf bis vierundzwanzig Jahren von zumeist jugendlichen Sexualtätern ermordet wurden. Der Schwerpunkt dieser Gruppe liegt auf den „Schutzlosen Ordensschwestern und Frauen gegen Ende des Zweiten Weltkrieges“, wobei das Andenken an 55 Ordensfrauen und 20 andere Frauen, meist aus den ehemaligen deutschen Ostgebieten, festgehalten ist. Angefügt ist eine Zusammenstellung der „Getöteten Beschützer bzw. Beschützerinnen der bedrohten Frauen gegen Ende des Zweiten Weltkrieges“. Von ihnen sind die Mehrzahl Priester, aber auch ein Vater, eine Hausfrau und zwei Ordensfrauen sind darunter. Der südwestdeutsche Raum ist in dieser Gruppe mit zwei dreizehnjährige Schülerinnen vertreten, die das Opfer jugendlicher Sexualmörder geworden sind: Cäcilia Baumann aus Zell am Harmersbach, die 1905 ermordet wurde, und Maria Grimm aus Winzingen, Kr. Göppingen, die 1922 ihren brutalen Tod fand.

Diese Opfer brutaler Sexualmorde stellt der Herausgeber unter dem Sammelbegriff „Reinheitsmartyrien“ zusammen und erinnert in der Einleitung an das Schicksal von Maria Goretti (1890-1902), ein elfjähriges Mädchen aus dem süditalienischen Nettuno, das nach der Überlieferung „lieber ... rein sterben, als sündigen“ wollte. „Sie [die Opfer] hatten etwas von der unverbrauchten Schönheit des ganzheitlich auf Gott allein hin bezogenen Lebens gespürt, die sie um keinen Preis verlieren wollten. Ihr Martyrium besteht darin, daß sie dem Gebot Gottes bis zum Äußersten gehorchen wollten, auch wenn es ihr junges Leben kosten sollte ${ }^{\text {*3 }}$. Die Angst, die Reinheit oder die Jungfräulichkeit zu verlieren, wurde damals den kleinen Mädchen in der Vorbereitung auf den Empfang der heiligen Kommunion ein-

3 Theologische Einführung zu: Zeugen für Christus (wie Anm. 1), Bd. 1, S. XLIII. 
geflößt. Die Deutung des Sexualmordes an der elfjährigen Maria Goretti dürfte vor diesem Hintergrund zu verstehen sein.

Der Breslauer Konsistorialrat Johannes Kaps hat nach dem Zweiten Weltkrieg die Leiden katholischer Frauen in Ostdeutschland in mehreren Publikationen dokumentiert ${ }^{4}$, die in das vorliegende Martyrologium aufgenommen wurden. Kaps stellte alle Frauen und Mädchen, die beim Einbruch der russischen Heere vergewaltigt wurden, als „Martyrerinnen der Reinheit“ dar. Und er deutet deren Schicksal mit den Worten, die Papst Pius XII. am Tag der Heiligsprechung der Maria Goretti am 24. Juni 1950 emphatisch auf dem Petersplatz äußerte: Es sei eine „liebevolle Fügung der göttlichen Vorsehung“, dass „einer bescheidenen Tochter des Volkes ... die höchste Ehrung“ der Heiligsprechung zu Teil werde. Durch den „blendenden Glanz und den berauschenden Wohlgeruch dieser Lilie im Purpurgewande“ [sei] bewirkt worden, dass sie soeben „mit innigster Freude in das Verzeichnis der Heiligen eingetragen“" wurde. In den Menschen, die sich aus Anlass der Heiligsprechung von Maria Goretti auf dem Petersplatz versammelt hatten, sah der Papst eine „Volksversammlung für die Reinheit“, eine Verschwörung gegen die Unsittlichkeit, die sich „der Bücher, der Illustrationen, der Schauspiele, der Hörfolgen, der Mode, der Strandbäder, der Vereine bedient“. Die Jugend forderte er auf, jedem Angriff gegen die Reinheit zu widerstehen.

Die Väter und Mütter verpflichtete er, ihre Söhne und Töchter zu schützen vor so vielen Gefahren, die sie umgeben, und sie von den Orten fernzuhalten, wo sie zu Gottlosigkeit und sittlichem Verderben verführt werden. Schließlich sprach der Papst die Heilige direkt an: „Sei gegrüßt, o süße und liebenswürdige Heilige! Martyrin auf Erden und Engel im Himmel, wende von Deiner Herrlichkeit Dein Auge diesem Volke zu, das Dich liebt, das Dich verehrt, das Dich verherrlicht, das Dich erhebt! Auf Deiner Stirne trägst Du hell und leuchtend den siegreichen Namen Christi (vgl. Apk 3, 12); auf Deinem jungfräulichen Antlitze erstrahlt die Kraft der Liebe und die Standhaftigkeit der Treue zum göttlichen Bräutigam; Du bist Blutbraut ...; Dir, die mächtig ist beim Lamme Gottes, vertrauen Wir diese Unsere hier gegenwärtigen Söhne und Töchter an und alle anderen, die im Geiste mit uns vereint sind. Sie bewundern Dein Heldentum, wollen aber noch mehr auch Deine Nachahmer sein im Eifer des Glaubens und in der unversehrbaren Reinheit der Sitten“"5.

Es ist wohl nicht übertrieben, zu behaupten, dass die meisten Beiträge der Gruppe $\mathrm{C}$ in diesem Martyrologium unter solch unrealistischen, aber pädagogisch relevanten Gesichtspunkten geschrieben wurden. Man will ja „den kommenden Gene-

${ }^{4}$ Martyrium und Heldentum Ostdeutscher Frauen. Ein Ausschnitt aus der schlesischen Passion 1945/46, bearb. und hg. von Johannes Kaps. Als Manuskript gedruckt. Nur für den Dienstgebrauch, München 1954.

${ }^{5}$ Kaps (wie Anm.4) S. 10-13; zitiert nach Kirchliches Amtsblatt für die Diözese Mainz, Nr. 15 vom 15. 8.1950, S. 93/94. Italienischer Text: Osservatore Romano, Nr. 149 vom 26./27. 6.1950 . 
rationen eine wirkmächtige Ermutigung an die Hand geben“, hieß es in der Einleitung. Heiligenviten, Heiligenlegenden und Verfahren, die Selig- und Heiligsprechungen vorbereiten, haben nicht das Interesse aufzuzeigen, wie man unter den geschichtlichen Bedingungen „heilig“ wird. Sie demonstrieren das Ideal dessen, was die Institution unter „heilig“ versteht. Zu diesem Zweck wird die Geschichte instrumentalisiert.

Angesichts der Massenvergewaltigung von Frauen am Kriegsende, vor allem in den Ostprovinzen des ehemaligen Deutschen Reiches, ist es im Hinblick auf die „Opfer“ wenig hilfreich, nach Jungfrauen, „gottgeweihten“ Jungfrauen und verheirateten Frauen zu unterscheiden. Sinnvoller wäre es für eine Kirche, die sich in der Nachfolge Jesu weiß, zu fragen, was ist mit den Frauen - ob Jungfrauen oder nicht -, die vergewaltigt wurden und die überlebt haben, geschehen? Hat man sich um sie und die Kinder, die sie ausgetragen haben, gekümmert?

Johannes Kaps hat in seiner Dokumentation Aussagen von Frauen überliefert, die am eigenen Leibe das Schicksal von Vergewaltigungen erfahren hatten. Sie wollten am liebsten über ihr Schicksal schweigen. Eine von ihnen berichtet: „Für mich ist es jetzt noch nach fünf Jahren sehr peinlich, darüber zu sprechen und regt mich noch immer sehr auf. Dann wurden darüber schon soviel Berichte geschrieben, geholfen hat es uns Mädeln doch nichts mehr, höchstens wurden wir von der Seite angesehen. Es ist wohl das Beste, darüber zu schweigen, denn die Unschuld bekommen wir doch nicht wieder"6.

In einem anderen Bericht heißt es: „Wer aber beugt sich vor dem Opfer der deutschen Frau des deutschen Ostens, die, geschändet, gequält, fürs Leben krank und siechend noch heute leben muß? Wer ehrt die Frau, die die Frucht solch weher und schrecklicher Stunden austrug und ein Kind gebar, das sie nicht wollte? Wer weiß um die Qual der Mutter, die vor ihren Kindern unzählige Male entehrt, die Liebe und das Zutrauen der eigenen Kinder verlor? Wer weiß um den Tod und das Erstarren allen Empfindens der Frauen und Mädchen, die in einer Nacht das Opfer von 40 bis 60 Russen waren und doch weiter leben müssen? Wer ahnt die Qual um das Zerreißen einer wahren, reinen und keuschen Mädchen- und Frauenseele? Wer begreift das Furchtbare, das unseren kleinen Mädchen angetan wurde? Unseren vierbis vierzehnjährigen? Sie leben noch meistens. Es gibt im Letzten kein Wort für das Leid der deutschen Frau im Osten“7.

Fragen von Überlebenden wurden in der Nachkriegszeit von den Verantwortlichen der Kirche kaum zufriedenstellend beantwortet. Sie deuteten die Situation und die Erfahrungen dieser Frauen „theologisch“, sie erörterten moraltheologische Prinzipien. Die Diskussion wurde ausschließlich von Männern und unter dem Gesichtspunkt der Sünde geführt. Es ging nicht um die Verletzung von Menschen-

${ }^{6}$ Kaps (wie Anm. 4) S. 15.

7 Aus dem Bericht einer Frau aus Liegnitz (Einzelbericht vom Juli 1951), deren Schwester, die Lehrerin Elisabeth J., am 19. April 1945 von den Russen erstochen wurde. Elisabeth J. war am 9. Februar 1914 geboren worden. Vgl. Kaps (wie Anm. 4) S. 84 f. 
rechten und Frauenwürde, es ging nicht um Verbrechen von Männern an Frauen, sondern um den Verlust der „Jungfräulichkeit“, um schließlich festzustellen, dass im Anschluss an den Kirchenvater Aurelius Augustinus „Jungfräulichkeit“ im theologischen Sinne weit mehr darstellt als die bloße anatomische Unverletztheit. Als geistig-sittliche Haltung bleibt sie, die Jungfräulichkeit, gewahrt trotz gewaltsamer physischer Deflorierung ${ }^{8}$.

Natürlich ist man sich der Sinnlosigkeit des Vergewaltigens und des Mordens schutzloser Frauen bewusst, aber auch dafür gibt es eine Erklärung, die letztlich dem Image der Institution Kirche zugute kommt. Wieder sind es theologische Begriffe, die oft eine lange religionsgeschichtliche Tradition vorweisen können, die auch in einem „aufgeklärten“ und „demokratischen“ Zeitalter ihre Wirkung zeigen. Man sprich von „Opfer“ und „Sühne“. Diese Begriffe werden unbesehen ihres magischen und mythischen Ursprungs in biblischen Texten eins zu eins ins Heute übertragen.

Ein Text auf dem Schutzumschlag zu dem Buch „Martyrium und Heldentum Ostdeutscher Frauen“ von Johannes Kaps hat seine Wirkung in den Nachkriegsjahren (und heute?) nicht verfehlt: „Asiatische Sturmgewalten tauchten Schlesien 1945 in apokalyptische Finsternis. Die Menschen dieser Landschaft schienen der Vernichtung preisgegeben. Die Verteidiger verzehrten ihre letzten Kräfte. Eine aufgepeitschte Soldateska erbrach die Tore und Türen des letzten Heims. Ihr Vernichtungswille zerstörte nicht nur das Menschenwerk dieser blühenden Landschaft, sondern vergiftete auch die Brunnen und Quellen alles menschlichen Lebens, indem sie die Frauen zum gejagten und erjagten Wild machten, zur Beute für ihr tierisches Gelüste. Aber mitten in diesem Vernichtungswahn standen Frauen und Mädchen auf, umgürteten sich mit dem Schwert der Reinheit und brachen den Ansturm der Feinde. Sie retteten die Zukunft ihres Volkes in ihrem Willen zur Keuschheit und gaben in Widerstand und Tod aus ihrer Seele Kraft von jenem Leben weiter, aus dem das schlesische und deutsche Volk wieder ein christliches Abendland bauen können. Der Wille dieser Frauen und Mädchen war stärker als der ihrer Feinde. Ihr Martyrium ist würdig dem Blutzeugnis der christlichen Frauen und Jungfrauen aller Jahrhunderte, die um ihrer Reinheit willen in den Tod gegangen sind“9

In den Texten, die das Schicksal der Frauen wiedergeben, ist viel von „Opfer“ und „Sühne“ die Rede. Die meisten Texte stammen aus der Feder von Männern. Nicht die Verbrechen der Männer, nicht die Sinnlosigkeit und die zerstörerische Gewalt der Kriege werden gebrandmarkt, vielmehr werden durch den Spruch der Kirche schutzlose und vergewaltigte und ermordete Frauen zu Helden und Heiligen stilisiert. Mit theologischen Begriffen werden die schrecklichen Erfahrungen und Leiden der Frauen letztlich instrumentalisiert, um die Institution Kirche zu

${ }^{8}$ Kaps (wie Anm. 4) S. 16 zitiert die entsprechenden moraltheologischen Autoritäten.

9 Ebd., Schutzumschlag (innen). 
verherrlichen. Für Johannes Paul II. ist das Martyrium der Frauen „ein leuchtendes Zeichen der Heiligkeit der Kirche“.

In ihrem ersten gemeinsamen Hirtenbrief nach dem Krieg im August 1945 mahnen die deutschen Bischofe eindringlich, dass nach Vergewaltigungen nichts gegen das keimende Leben unternommen werden darf: „Leben darf auch dann nicht bewußt und gewollt vernichtet werden, wenn es durch Betrug oder Vergewaltigung seinen Ursprung genommen hat. Rein menschlich gesehen, gehören solche Fälle sicher zu den tragischsten, in die eine Frau geraten kann, und es gehört eine große sittliche Kraft dazu, dieses Leid durchzustehen. Alle, die mit solchen Fällen in Berührung kommen, ... werden mit größter Anteilnahme der Unglücklichen sich annehmen und ihr Los zu erleichtern suchen. Es werden mit öffentlicher Hilfe nötigenfalls Anstalten gegründet ... in denen solche Kinder aufgenommen werden können, um in christlicher Liebe erzogen zu werden. Niemals aber kann es gestattet sein und niemand kann das Recht geben, in die Herrscherrechte Gottes einzugreifen und das keimende Leben zu töten! .... Die Mütter werden, so schwer es ihnen fällt, bemüht sein müssen, weniger an das ihnen widerfahrene Unrecht, als an das unschuldige Leben zu denken, das heranreift. Gott, vor dessen Auge sie schuldlos dastehen, wird ihnen die Kraft schenken, ihr Los in christlichem Starkmut zu tragen ..."10.

Fassen wir diesen Punk zusammen: Mit theologischen Begriffen ist diese bittere Realität nicht zu fassen. Äußerungen des kirchlichen Lehramtes oder auch Ansprachen der Päpste und der meisten Bischöfe nehmen die gefühlte Wirklichkeit nicht wahr. Die Menschen an der Basis der Kirche sind nicht immer kritisch genug, die Sprache der Amtskirche zu durchschauen. Weil diesen Worten, die meist zu nichtssagenden Formeln erstarrt sind, nicht widersprochen wird, können sie zur Stabilisierung der Macht (der Eliten, des Amtes) beitragen. Das Frauenbild vor allem, aber auch das Menschen- und Familienbild der Amtskirche ist bis heute von diesen archaischen Vorstellungen dominiert. Aus der heutigen, gesellschaftlich gängigen Sicht muss man sagen: Frauen werden weiterhin diskriminiert.

Vielleicht müsste man die kritische Sicht, die wir im Zusammenhang der „Reinheitsmartyrien“ gewonnen haben, auf die gesamte Edition „Zeugen für Christus“ anwenden, mit Sicherheit aber auf die Gruppe D: „Blutzeugen aus den Missionsgebieten“. Das Missionsverständnis hat sich in den letzten 50 Jahren gewandelt. Nicht dass man die Martyrer und Martyrerinnen wegen ihres „Missionseifers“, der in der Zeit des Kolonialismus ausgebildet wurde, verurteilen müsste, aber die Sprache der Darstellung heute müsste kritischer sein, denn das Umfeld der christlichen Mission in Asien zum Beispiel ist von wirtschaftlichen Machtkämpfen und kriegerischen Auseinandersetzungen geprägt. Mit dem Angriff der Kaiserlich-Japanischen Marineluftstreitkräfte auf die amerikanische Pazifikflotte in Pearl Harbor am 7. De-

10 Gemeinsamer Hirtenbrief der deutschen Bischöfe vom 23. August 1945, zitiert bei Kaps (wie Anm. 4) S. $18 \mathrm{f}$. 
zember 1941 begann die japanische Offensive gegen die britischen und niederländischen Kolonien in Südostasien. Der Krieg in Europa weitete sich zu einem Weltkrieg aus. Christliche Missionsstationen, Mitglieder katholischer europäischer Missionsorden, Pater, Brüder und Schwestern wurden in den von den Japanern eroberten und besetzten Gebieten in den Strudel der Gewalt hineingezogen. Sie wurden Opfer von Massakern japanischer Soldaten und von Kriegsverbrechen der amerikanischen Luftwaffe. Man ist geneigt, in ihnen Opfer des Kolonialismus zu sehen.

Zum Teil sind die geschichtlichen Fakten durch die Berichte der einzelnen Biografien belegt. So wurde zum Beispiel eine Gruppe Steyler Missionare, meistens deutsche Staatsangehörige, unter ihnen der Missionsbischof Joseph Lörks, Patres, Brüder und Schwestern, außerdem sechs weitere Personen, Angehörige der Liebenzeller Mission, Mitglieder der Herz-Jesu-Missionare, Missionsschwestern desselben Ordens und mehrere nichtdeutsche Europäer, insgesamt 58 Personen, am 17. März 1944 auf dem japanischen Kriegsschiff „Akikaze“ auf offener See von japanischen Militärs erschossen und ins Meer versenkt. Sie waren auf Zentral NeuGuinea von den Japanern im Dezember 1942 interniert worden. Die Japaner verdächtigten sie der Spionage für die Amerikaner und Australier.

Eine andere Gruppe Steyler Missionare, Patres, Brüder und Schwestern, Mitglieder der Gesellschaft des Göttlichen Wortes und amerikanische lutherische Missionare, die im Norden von Neu-Guinea tätig waren, wurden ebenfalls von den Japanern interniert. Sie sollten auf der „Yorishime Maru“, einem japanischen Handelsschiff, nach Hollandia in Niederländisch-Guinea gebracht werden. Am 6. Februar 1944 nahmen elf amerikanische Flugzeuge das Handelsschiff unter Beschuss. Mehr als 60 Personen wurden unmittelbar getötet oder verstarben innerhalb der nächsten Wochen.

Ein letztes Beispiel: In dem Kolleg der Schulbrüder in Manila, die auf eine dreißigjährige erfolgreiche Tätigkeit auf den Philippinen zurückblicken konnten, richteten die japanischen Soldaten am 12. Februar 1945 ein schreckliches Massaker an, bei dem 17 Schulbrüder, aber auch Zivilisten, Angestellte des Kollegs, auf grausame Weise umgebracht wurden. Unter anderem wird berichtet: Die Soldaten, deren Augen blutunterlaufen waren, einige von ihnen waren betrunken, fingen an, auf alle, derer sie habhaft werden konnten, zu schießen oder mit dem aufgepflanzten Seitengewehr einzustechen.

„Blutzeugen aus den Missionsgebieten“, Ordensmänner und Ordensfrauen, die aus dem Südwesten Deutschlands stammen, sind folgende: P. Franziskus (Karl) Leutner OSB aus Friesenheim bei Lahr, P. Hermenegild (Karl) Wäldele OFM aus Oberkirch/Baden, Franziskanerin Sr. M. Benigna (Maria) Schweizer aus Deggingen, Kr. Göppingen, P. Konrad (Friedrich) Rapp OSB aus Elzach/Baden, Steyler Missionsschwester Ubaldine (Maria) Henkel aus Bernhofen bei Ravensburg, Steyler Missionsbruder Jason (Willibald) Ziesel aus Bonlanden, Kr. Biberach, die Steyler Missionsschwestern Sr. Festina (Katharina) Blank aus Bildechingen bei Horb, 
Sr. Annette (Anna), Sr. Benerda (Magdalena) Kuhn aus Ramsenstrut bei Ellwangen, Sr. Milia (Emma) Schäfer aus Königsheim bei Tuttlingen, Annacrescenz (Mechthilde) Wehinger aus Aulfingen, Kr. Tuttlingen, die Schulbrüder Berthwin Philibert (Hermann Joseph) Gelb aus Hockenheim, Hartmann Hubert (Lorenz) Kreitner aus Mannheim, Friedebert-Johannes (Johannes) Kuntz aus Rheinfelden, Viktorinus Heinrich (Konrad) Wehle aus Grünmettstetten bei Horb, P. Gregor (Ludwig Karl Friedrich) Sorger OSB aus Spaichingen, P. Anselm (Josef) Romer aus Ingerkingen bei Laupheim, P. Arnulf (Josef) Schleicher aus Pflaumloch bei Neresheim, die Trappisten-Brüder Severin (Josef) Boos aus Dunstelkingen, Modestus (August) Hipper aus Unterstadion, Remigius (Franz) Kraus aus Bruchsal und Gaudentius (Gregor) Rupp aus Dorfmerkingen, die Steyler Missionare P. August Hättig aus Kuhbach bei Lahr und P. Richard Haas aus Schutterwald.

Männer und Frauen, die in allen vier Gruppen aufgelistet und zumeist mit Kurzbiografien und ihren Leidensgeschichten vorgestellt werden, sind zum größten Teil die Opfer totalitärer Systeme, brutaler Gewalt und vernichtender und sinnloser Kriege. Wie immer sie die letzten Augenblicke ihres Lebens erfahren haben, ihnen allen ist gemeinsam die elementare Abwehr gegenüber der Unmenschlichkeit, mit der sie die Täter attackiert haben. Die Masse der Täter ließ sich von den Herrschenden und ihren Helfershelfern instrumentalisieren oder missbrauchen.

Die Namen der Opfer sollten nicht vergessen werden. Die Erinnerung an sie, an jedes einzelne Schicksal, das sinnlos vernichtet wurde, sollte künftige Generationen sensibilisieren. Sie sollen erkennen, dass die Ursachen des menschenverachtenden und menschenvernichtenden Handelns in egoistisch-nationalistischem Denken, in absoluten Wahrheitsansprüchen totalitärer Herrschaftssysteme, in jeglichem ideologisch begründeten Aus- und Abgrenzen (die Kirche macht da keine Ausnahme) und im Denken in Feindbildern liegen. Natürlich muss auch beachtet werden, dass hinter all den Grausamkeiten Machtansprüche und zumeist wirtschaftliche Interessen stehen. Bei den pädagogischen Aufrufen, die Martyrer zu verehren, kommt dieser historisch belegbare Aspekt nur selten zur Geltung.

Trotz allem ist die Auflistung der Namen, mögen die Recherchen noch so mühsam sein, ein notwendiges und wichtiges Unternehmen. Aber es würde genügen, dies als „Nekrolog/Totenlisten der Opfer von Gewalt und Krieg“ zu bezeichnen. Wenn dann andere gesellschaftliche Gruppen oder Konfessionen solche Listen erstellten, wäre eine Barriere der Abgrenzung schon überschritten und ein wichtiger Schritt in Richtung Solidarisierung der Menschen untereinander getan. Die Solidarisierung, die im Leben oft nicht zustande gekommen ist, wäre vielleicht für die Zukunft vorbereitet.

Bedauerlicherweise werden in dem Fall, in dem durch Widerstand gegen das Gewaltsystem die konfessionellen Abgrenzungen überwunden wurden, wie bei dem Freundeskreis „Weiße Rose“, nur die katholischen Mitglieder mit einer eigenen Biografie gewürdigt, nämlich der Münchner Universitätsprofessor Kurt Huber, der Medizinstudent Willi Graf und der Medizinstudent Christoph Probst. Probst 
war konfessionslos aufgewachsen, war über seinen Freund, den im orthodoxen Glauben erzogenen Alexander Schmorell, mit dem Christentum in Berührung gekommen und ließ sich nach der Urteilsverkündigung im Gefängnis katholisch taufen. Das genügte, damit seine Biografie in das Martyrologium aufgenommen wurde. Alexander Schmorell wurde inzwischen von der orthodoxen Kirche heiliggesprochen. Seine Biografie hätte gut in diesen Sammelband gepasst. Sein Name wird etwas verschämt zusammen mit den Namen Sophie und Hans Scholl im Personenregister unter einer eigenen Rubrik, die es allerdings nur im Register gibt, erwähnt. Die Seitenangaben, die sich auf die Biografien der katholischen Mitglieder der „Weißen Rose“ beziehen, belegen, dass es im Widerstand ein gemeinsames, Konfessionsgrenzen sprengendes Interesse gab. Ähnlich verhält es sich mit den Namen des evangelischen Theologen Dietrich Bonhoeffer und des Pastors an der Luther-Kirche in Lübeck, Karl Friedrich Stellbrink. Obwohl Stellbrink sich dem Widerstand der katholischen Geistlichen in Lübeck, mit Johannes Prassek, Eduard Müller und Hermann Lange, angeschlossen hatte und sie gemeinsam im „Lübecker Christenprozess“ zum Tode verurteilt wurden, war es zu Beginn des 21. Jahrhunderts nicht möglich, ihn und die anderen erwähnten Protestanten in einer von der Deutschen Bischofskonferenz finanzierten Publikation mit einer Biografie zu würdigen.

Unter dem Aspekt des sich Erinnerns an die Opfer von Gewalt und Krieg sollen die Interessen der Leser dieser Zeitschrift geweckt werden. Wirksamer ist es, sich nicht nur der Opfer allgemein zu erinnern: Wenn man weiß, dieser Mann, jene Frau haben in meiner Nähe gelebt, sie sind in meiner engeren Umgebung geboren, dann kann man Empathie aufbringen und dazu beitragen, dass sich solche Katastrophen wie unter der Diktatur totalitärer Staaten nie mehr ereignen. Auch wenn Lenin mit seinem Spruch „die Wahrheit ist konkret“ etwas anderes meinte, hier gilt er. 